PROCEEDINGS OF THE

AMERICAN MATHEMATICAL SOCIETY

Volume 127, Number 9, Pages 2657-2664

S 0002-9939(99)05285-5

Article electronically published on April 23, 1999

\title{
WEIGHTED CACCIOPPOLI-TYPE ESTIMATES AND WEAK REVERSE HÖLDER INEQUALITIES FOR $A$-HARMONIC TENSORS
}

\author{
SHUSEN DING
}

(Communicated by Christopher D. Sogge)

\begin{abstract}
We obtain a local weighted Caccioppoli-type estimate and prove the weighted version of the weak reverse Hölder inequality for $A$-harmonic tensors.
\end{abstract}

\section{INTRODUCTION}

Harmonic functions have wide applications in many fields, such as potential theory, partial differential equations, harmonic analysis and the theory of $H^{p}$-spaces. $A$-harmonic tensors are interesting and important generalizations of $p$-harmonic tensors. In the meantime, $p$-harmonic tensors are extensions of conjugate harmonic functions and $p$-harmonic functions, $p>1$. In recent years there have been remarkable advances made in the field of $A$-harmonic tensors. Many interesting results of $A$-harmonic tensors and their applications in fields such as potential theory, quasiregular mappings and the theory of elasticity have been found; see [1], [2], [3], [7], [8], [9], [10], [11], [12], [14]. For many purposes, we need to know the integrability of $A$-harmonic tensors and estimate the integrals for $A$-harmonic tensors. In this paper we first obtain the local weighted Caccioppoli-type estimate and the weighted version of the weak reverse Hölder inequality for $A$-harmonic tensors. These integral inequalities can be used to study the integrability of $A$-harmonic tensors and estimate the integrals for $A$-harmonic tensors.

We always assume $\Omega$ is a connected open subset of $\mathbf{R}^{n}$ throughout this paper. Let $e_{1}, e_{2}, \cdots, e_{n}$ denote the standard unit basis of $\mathbf{R}^{n}$. For $l=0,1, \cdots, n$, the linear space of $l$-vectors, spanned by the exterior products $e_{I}=e_{i_{1}} \wedge e_{i_{2}} \wedge \cdots \wedge e_{i_{l}}$, corresponding to all ordered $l$-tuples $I=\left(i_{1}, i_{2}, \cdots, i_{l}\right), 1 \leq i_{1}<i_{2}<\cdots<i_{l} \leq n$, is denoted by $\wedge^{l}=\wedge^{l}\left(\mathbf{R}^{n}\right)$. The Grassmann algebra $\wedge=\bigoplus \wedge^{l}$ is a graded algebra with respect to the exterior products. For $\alpha=\sum \alpha^{I} e_{I} \in \wedge$ and $\beta=\sum \beta^{I} e_{I} \in \wedge$, the inner product in $\wedge$ is given by $\langle\alpha, \beta\rangle=\sum \alpha^{I} \beta^{I}$ with summation over all $l$ tuples $I=\left(i_{1}, i_{2}, \cdots, i_{l}\right)$ and all integers $l=0,1, \cdots, n$. We define the Hodge star operator $\star: \wedge \rightarrow \wedge$ by the rule $\star 1=e_{1} \wedge e_{2} \wedge \cdots \wedge e_{n}$ and $\alpha \wedge \star \beta=\beta \wedge \star \alpha=\langle\alpha, \beta\rangle(\star 1)$ for all $\alpha, \beta \in \wedge$.

Received by the editors August 23, 1997.

1991 Mathematics Subject Classification. Primary 30C65; Secondary 31B05, 58A10.

Key words and phrases. A-harmonic tensors, $A_{r}$-weights, Caccioppoli-type estimate, $A$ harmonic equation. 
Hence the norm of $\alpha \in \wedge$ is given by the formula $|\alpha|^{2}=\langle\alpha, \alpha\rangle=\star(\alpha \wedge \star \alpha) \in \wedge^{0}=$ R. The Hodge star is an isometric isomorphism on $\wedge$ with $\star: \wedge^{l} \rightarrow \wedge^{n-l}$ and $\star \star(-1)^{l(n-l)}: \wedge^{l} \rightarrow \wedge^{l}$. Let $0<p<\infty$; we denote the weighted $L^{p}$-norm of a measurable function $f$ over $E$ by

$$
\|f\|_{p, E, w}=\left(\int_{E}|f(x)|^{p} w(x) d x\right)^{1 / p} .
$$

A differential $l$-form $\omega$ on $\Omega$ is a Schwartz distribution on $\Omega$ with values in $\wedge^{l}\left(\mathbf{R}^{n}\right)$. We denote the space of differential $l$-forms by $D^{\prime}\left(\Omega, \wedge^{l}\right)$. We write $L^{p}\left(\Omega, \wedge^{l}\right)$ for the $l$-forms $\omega(x)=\sum_{I} \omega_{I}(x) d x_{I}=\sum \omega_{i_{1} i_{2} \cdots i_{l}}(x) d x_{i_{1}} \wedge d x_{i_{2}} \wedge \cdots \wedge d x_{i_{l}}$ with $\omega_{I} \in L^{p}(\Omega, \mathbf{R})$ for all ordered $l$-tuples $I$. Thus $L^{p}\left(\Omega, \wedge^{l}\right)$ is a Banach space with norm

$$
\|\omega\|_{p, \Omega}=\left(\int_{\Omega}|\omega(x)|^{p} d x\right)^{1 / p}=\left(\int_{\Omega}\left(\sum_{I}\left|\omega_{I}(x)\right|^{2}\right)^{p / 2} d x\right)^{1 / p} .
$$

Similarly, $W_{p}^{1}\left(\Omega, \wedge^{l}\right)$ are those differential $l$-forms on $\Omega$ whose coefficients are in $W_{p}^{1}(\Omega, \mathbf{R})$. The notations $W_{p, l o c}^{1}(\Omega, \mathbf{R})$ and $W_{p, l o c}^{1}\left(\Omega, \wedge^{l}\right)$ are self-explanatory. We denote the exterior derivative by $d: D^{\prime}\left(\Omega, \wedge^{l}\right) \rightarrow D^{\prime}\left(\Omega, \wedge^{l+1}\right)$ for $l=0,1, \cdots, n$. Its formal adjoint operator $d^{\star}: D^{\prime}\left(\Omega, \wedge^{l+1}\right) \rightarrow D^{\prime}\left(\Omega, \wedge^{l}\right)$ is given by $d^{\star}=(-1)^{n l+1} \star d \star$ on $D^{\prime}\left(\Omega, \wedge^{l+1}\right), l=0,1, \cdots, n$.

Recently there has been new interest developed in the study of the $A$-harmonic equation for differential forms, largely pertaining to applications in quasiconformal analysis and nonlinear elasticity, that is:

$$
d^{\star} A(x, d \omega)=0,
$$

where $A: \Omega \times \wedge^{l}\left(\mathbf{R}^{n}\right) \rightarrow \wedge^{l}\left(\mathbf{R}^{n}\right)$ satisfies the following conditions:

$$
|A(x, \xi)| \leq a|\xi|^{p-1} \text { and }\langle A(x, \xi), \xi\rangle \geq|\xi|^{p}
$$

for almost every $x \in \Omega$ and all $\xi \in \wedge^{l}\left(\mathbf{R}^{n}\right)$. Here $a>0$ is a constant and $1<p<\infty$ is a fixed exponent associated with (1.1). A solution to (1.1) is an element of the Sobolev space $W_{p, l o c}^{1}\left(\Omega, \wedge^{l-1}\right)$ such that

$$
\int_{\Omega}\langle A(x, d \omega), d \varphi\rangle=0
$$

for all $\varphi \in W_{p}^{1}\left(\Omega, \wedge^{l-1}\right)$ with compact support.

Definition 1.3. We call $u$ an $A$-harmonic tensor in $\Omega$ if $u$ satisfies the $A$-harmonic equation (1.1) in $\Omega$.

Let us mention some basic terms for harmonic tensors as follows. A differential $l$-form $u \in D^{\prime}\left(\Omega, \wedge^{l}\right)$ is called a closed form if $d u=0$ in $\Omega$. A differential form $u$ is called a $p$-harmonic tensor if

$$
d^{\star}\left(|d u|^{p-2} d u\right)=0 \text { and } d^{\star} u=0,
$$

where $1<p<\infty$. See [7] for more results about $p$-harmonic tensors. In order to formulate some estimates it is required first of all that the equation be written in the form of a first order differential system:

$$
A(x, d u)=d^{\star} v .
$$


In this way we obtain a pair $(u, v)$ of $(l-1)$-form $u$ and $(l+1)$-form $v$, called the conjugate $A$-harmonic fields. Example: $d u=d^{*} v$ is an analogue of a CauchyRiemann system in $\mathbf{R}^{n}$. Clearly, the $A$-harmonic equation is not affected by adding a closed form to $u$ and coclosed form to $v$. Therefore, any type of estimates between $u$ and $v$ must be modulo such forms. Suppose that $u$ is a solution to (1.1) in $\Omega$. Then, at least locally in a ball $B$, there exists a form $v \in W_{q}^{1}\left(B, \wedge^{l+1}\right), \frac{1}{p}+\frac{1}{q}=1$, such that (1.4) holds.

Definition 1.5. When $u$ and $v$ satisfy (1.4) in $\Omega$, and $A^{-1}$ exists in $\Omega$, we call $u$ and $v$ conjugate $A$-harmonic tensors in $\Omega$.

Definition 1.6. We call $u$ a $p$-harmonic function if $u$ satisfies the $p$-harmonic equation

$$
\operatorname{div}\left(\nabla u|\nabla u|^{p-2}\right)=0
$$

with $p>1$. Its conjugate in the plane is a $q$-harmonic function $v, p^{-1}+q^{-1}=1$, which satisfies

$$
\nabla u|\nabla u|^{p-2}=\left(\frac{\partial v}{\partial y},-\frac{\partial v}{\partial x}\right)
$$

Note that if $p=q=2$, we get the usual conjugate harmonic functions.

We write $\mathbf{R}=\mathbf{R}^{1}$. Balls are denoted by $B$ and $\sigma B$ is the ball with the same center as $B$ and with $\operatorname{diam}(\sigma B)=\sigma \operatorname{diam}(B)$. The $n$-dimensional Lebesgue measure of a set $E \subseteq \mathbf{R}^{n}$ is denoted by $|E|$. We call $w$ a weight if $w \in L_{l o c}^{1}\left(\mathbf{R}^{n}\right)$ and $w>0$ a.e. Also in general $d \mu=w d x$ where $w$ is a weight. The following result appears in [8]: Let $Q \subset \mathbf{R}^{n}$ be a cube or a ball. To each $y \in Q$ there corresponds a linear operator $K_{y}: C^{\infty}\left(Q, \wedge^{l}\right) \rightarrow C^{\infty}\left(Q, \wedge^{l-1}\right)$ defined by

$$
\left(K_{y} \omega\right)\left(x ; \xi_{1}, \cdots, \xi_{l}\right)=\int_{0}^{1} t^{l-1} \omega\left(t x+y-t y ; x-y, \xi_{1}, \cdots, \xi_{l-1}\right) d t
$$

and the decomposition

$$
\omega=d\left(K_{y} \omega\right)+K_{y}(d \omega)
$$

We define another linear operator $T_{Q}: C^{\infty}\left(Q, \wedge^{l}\right) \rightarrow C^{\infty}\left(Q, \wedge^{l-1}\right)$ by averaging $K_{y}$ over all points $y$ in $Q$

$$
T_{Q} \omega=\int_{Q} \varphi(y) K_{y} \omega d y
$$

where $\varphi \in C_{0}^{\infty}(Q)$ is normalized by $\int_{Q} \varphi(y) d y=1$. We define the $l$-form $\omega_{Q} \in$ $D^{\prime}\left(Q, \wedge^{l}\right)$ by

$$
\omega_{Q}=|Q|^{-1} \int_{Q} \omega(y) d y, \quad l=0, \text { and } \omega_{Q}=d\left(T_{Q} \omega\right), \quad l=1,2, \cdots, n,
$$

for all $\omega \in L^{p}\left(Q, \wedge^{l}\right), 1 \leq p<\infty$.

\section{The local Weighted Caccioppoli-type estimate}

Definition 2.1. We say the weight $w(x)$ satisfies the $A_{r}$ condition, $r>1$, written $w \in A_{r}$, if $w(x)>0$ a.e., and, for any ball $B \subset \mathbf{R}^{n}$,

$$
\sup _{B}\left(\frac{1}{|B|} \int_{B} w d x\right)\left(\frac{1}{|B|} \int_{B}\left(\frac{1}{w}\right)^{1 /(r-1)} d x\right)^{(r-1)}<\infty .
$$


See [5] and [6] for the basic properties of $A_{r}$-weights. We need the following lemma [5].

Lemma 2.2. If $w \in A_{r}$, then there exist constants $\beta>1$ and $C$, independent of w, such that

$$
\|w\|_{\beta, B} \leq C|B|^{(1-\beta) / \beta}\|w\|_{1, B}
$$

for all balls $B \subset \mathbf{R}^{n}$.

We will also need the following generalized Hölder's inequality.

Lemma 2.3. Let $0<\alpha<\infty, 0<\beta<\infty$ and $s^{-1}=\alpha^{-1}+\beta^{-1}$. If $f$ and $g$ are measurable functions on $\mathbf{R}^{n}$, then

$$
\|f g\|_{s, \Omega} \leq\|f\|_{\alpha, \Omega} \cdot\|g\|_{\beta, \Omega}
$$

for any $\Omega \subset \mathbf{R}^{n}$.

In [10], C. A. Nolder obtains the following local Caccioppoli-type estimate.

Theorem A. Let $u$ be an A-harmonic tensor in $\Omega$ and let $\sigma>1$. Then there exists a constant $C$, independent of $u$ and $d u$, such that

$$
\|d u\|_{s, B} \leq C|B|^{-1}\|u-c\|_{s, \sigma B}
$$

for all balls or cubes $B$ with $\sigma B \subset \Omega$ and all closed forms $c$. Here $1<s<\infty$.

The following weak reverse Hölder inequality appears in [10].

Theorem B. Let $u$ be an A-harmonic tensor in $\Omega, \sigma>1$ and $0<s, t<\infty$. Then there exists a constant $C$, independent of $u$, such that

$$
\|u\|_{s, B} \leq C|B|^{(t-s) / s t}\|u\|_{t, \sigma B}
$$

for all balls or cubes $B$ with $\sigma B \subset \Omega$.

We now generalize Theorem A into the following local weighted Caccioppoli-type estimate for $A$-harmonic tensors.

Theorem 2.5. Let $u \in D^{\prime}\left(\Omega, \wedge^{l}\right), l=0,1, \cdots, n$, be an A-harmonic tensor in a domain $\Omega \subset \mathbf{R}^{n}$ and $\rho>1$. Assume that $1<s<\infty$ is a fixed exponent associated with the A-harmonic equation and $w \in A_{r}$ for some $r>1$. Then there exists a constant $C$, independent of $u$ and $d u$, such that

$$
\|d u\|_{s, B, w} \leq C|B|^{-1}\|u-c\|_{s, \rho B, w},
$$

for all balls $B$ with $\rho B \subset \Omega$ and all closed forms $c$.

Note that (2.6) can be written as

$$
\left(\int_{B}|d u|^{s} w d x\right)^{1 / s} \leq \frac{C}{|B|}\left(\int_{\rho B}|u-c|^{s} w d x\right)^{1 / s},
$$

Or

$$
\left(\int_{B}|d u|^{s} d \mu\right)^{1 / s} \leq \frac{C}{|B|}\left(\int_{\rho B}|u-c|^{s} d \mu\right)^{1 / s},
$$

where the measure $\mu$ is defined by $d \mu=w(x) d x$ and $w \in A_{r}$. 
Proof. Since $w \in A_{r}$ for some $r>1$, by Lemma 2.2, there exist constants $\beta>1$ and $C_{1}>0$, such that

$$
\|w\|_{\beta, B} \leq C_{1}|B|^{(1-\beta) / \beta}\|w\|_{1, B}
$$

for any cube or any ball $B \subset \mathbf{R}^{n}$. Choose $t=s \beta /(\beta-1)$; then $1<s<t$ and $\beta=t /(t-s)$. Since $1 / s=1 / t+(t-s) / s t$, by Hölder's inequality, Theorem A and (2.7), we have

$$
\begin{aligned}
\|d u\|_{s, B, w} & =\left(\int_{B}\left(|d u| w^{1 / s}\right)^{s} d x\right)^{1 / s} \\
& \leq\left(\int_{B}|d u|^{t} d x\right)^{1 / t}\left(\int_{B}\left(w^{1 / s}\right)^{s t /(t-s)} d x\right)^{(t-s) / s t} \\
& \leq C_{2}\|d u\|_{t, B} \cdot\|w\|_{\beta, B}^{1 / s} \\
& \leq C_{3}|B|^{-1}\|u-c\|_{t, \sigma B} \cdot\|w\|_{\beta, B}^{1 / s} \\
& \leq C_{4}|B|^{-1}|B|^{(1-\beta) / \beta s}\|w\|_{1, B}^{1 / s} \cdot\|u-c\|_{t, \sigma B} \\
& =C_{4}|B|^{-1}|B|^{-1 / t} \cdot\|w\|_{1, B}^{1 / s} \cdot\|u-c\|_{t, \sigma B}
\end{aligned}
$$

for all balls $B$ with $\sigma B \subset \Omega$ and all closed forms $c$. Since $c$ is a closed form and $u$ is an $A$-harmonic tensor, then $u-c$ is still an $A$-harmonic tensor. Taking $m=s / r$, we find that $m<s<t$. Applying Theorem B yields

$$
\begin{aligned}
\|u-c\|_{t, \sigma B} & \leq C_{5}|B|^{(m-t) / m t}\|u-c\|_{m, \sigma^{2} B} \\
& \leq C_{5}|B|^{(m-t) / m t}\|u-c\|_{m, \rho B}
\end{aligned}
$$

where $\rho=\sigma^{2}$. Substituting (2.9) in (2.8), we have

$$
\|d u\|_{s, B, w} \leq C_{6}|B|^{-1}|B|^{-1 / m} \cdot\|w\|_{1, B}^{1 / s} \cdot\|u-c\|_{m, \rho B} .
$$

Now $1 / m=1 / s+(s-m) / s m$; by Hölder's inequality again, we obtain

$$
\begin{aligned}
\|u-c\|_{m, \rho B} & =\left(\int_{\rho B}|u-c|^{m} d x\right)^{1 / m} \\
& =\left(\int_{\rho B}\left(|u-c| w^{1 / s} w^{-1 / s}\right)^{m} d x\right)^{1 / m} \\
& \leq\left(\int_{\rho B}|u-c|^{s} w d x\right)^{1 / s}\left(\int_{\rho B}\left(\frac{1}{w}\right)^{m /(s-m)} d x\right)^{(s-m) / s m} \\
& \leq\|u-c\|_{s, \rho B, w} \cdot\|1 / w\|_{m /(s-m), \rho B}^{1 / s}
\end{aligned}
$$

for all balls $B$ with $\rho B \subset \Omega$ and all closed forms $c$. Combining (2.10) and (2.11), we obtain

$$
\|d u\|_{s, B, w} \leq C_{6}|B|^{-1}|B|^{-1 / m} \cdot\|w\|_{1, B}^{1 / s} \cdot\|1 / w\|_{m /(s-m), \rho B}^{1 / s} \cdot\|u-c\|_{s, \rho B, w} .
$$


Since $w \in A_{r}$, we then have

$$
\begin{aligned}
&\|w\|_{1, B}^{1 / s} \cdot\|1 / w\|_{m /(s-m), \rho B}^{1 / s}=\left(\int_{B} w d x\right)^{1 / s}\left(\int_{\rho B}\left(\frac{1}{w}\right)^{m /(s-m)} d x\right)^{(s-m) / s m} \\
& \leq\left(\left(\int_{\rho B} w d x\right)\left(\int_{\rho B}\left(\frac{1}{w}\right)^{1 /(s / m-1)} d x\right)^{s / m-1}\right)^{1 / s} \\
&=\left(|\rho B|^{s / m}\left(\frac{1}{|\rho B|} \int_{\rho B} w d x\right)\left(\frac{1}{|\rho B|} \int_{\rho B}\left(\frac{1}{w}\right)^{1 /(r-1)} d x\right)^{r-1}\right)^{1 / s} \\
& \leq C_{7}|B|^{1 / m} .
\end{aligned}
$$

Substituting (2.13) in (2.12), we find that

$$
\|d u\|_{s, B, w} \leq C|B|^{-1}\|u-c\|_{s, \rho B, w}
$$

for all balls $B$ with $\rho B \subset \Omega$ and all closed forms $c$. This ends the proof of Theorem 2.5 .

\section{The WeIGHTED VERSION OF THE WEAK REVERSE HöLDER INEQUALITY}

We now generalize Theorem B into the following weighted form.

Theorem 3.1. Let $u \in D^{\prime}\left(\Omega, \wedge^{l}\right), l=0,1, \cdots, n$, be an A-harmonic tensor in a domain $\Omega \subset \mathbf{R}^{n}, \sigma>1$. Assume that $0<s, t<\infty$ and $w \in A_{r}$ for some $r>1$. Then there exists a constant $C$, independent of $u$, such that

$$
\left(\int_{B}|u|^{s} w d x\right)^{1 / s} \leq C|B|^{(t-s) / s t}\left(\int_{\sigma B}|u|^{t} w^{t / s} d x\right)^{1 / t}
$$

for all balls $B$ with $\sigma B \subset \Omega$.

The proof of Theorem 3.1 is similar to that of Theorem 2.5. For completion of the paper, we prove Theorem 3.1 as follows.

Proof. Since $w \in A_{r}$ for some $r>1$, by Lemma 2.2, there exist constants $\beta>1$ and $C_{1}>0$, such that

$$
\|w\|_{\beta, B} \leq C_{1}|B|^{(1-\beta) / \beta}\|w\|_{1, B}
$$

for any cube or any ball $B \subset \mathbf{R}^{n}$. Choose $k=s \beta /(\beta-1)$; then $s<k$ and $\beta=k /(k-s)$. By (3.3) and Hölder's inequality, we have

$$
\begin{aligned}
\|u\|_{s, B, w} & \leq\left(\int_{B}|u|^{k} d x\right)^{1 / k}\left(\int_{B}\left(w^{1 / s}\right)^{s k /(k-s)} d x\right)^{(k-s) / s k} \\
& =\|u\|_{k, B} \cdot\|w\|_{\beta, B}^{1 / s} \\
& \leq C_{2}|B|^{(1-\beta) / \beta s}\|w\|_{1, B}^{1 / s} \cdot\|u\|_{k, B} \\
& =C_{2}|B|^{-1 / k}\|w\|_{1, B}^{1 / s} \cdot\|u\|_{k, B}
\end{aligned}
$$


for all balls $B$ with $\sigma B \subset \Omega$. Choosing $m=s t /(s+t(r-1))$, by Theorem B we obtain

$$
\|u\|_{k, B} \leq C_{3}|B|^{(m-k) / k m}\|u\|_{m, \sigma B} .
$$

Combining (3.4) and (3.5) yields

$$
\|u\|_{s, B, w} \leq C_{4}|B|^{-1 / m}\|w\|_{1, B}^{1 / s} \cdot\|u\|_{m, \sigma B} .
$$

Since $m<t$, by Hölder's inequality, we have

$$
\begin{aligned}
\|u\|_{m, \sigma B} & =\left(\int_{\sigma B}\left(|u| w^{1 / s} w^{-1 / s}\right)^{m} d x\right)^{1 / m} \\
& \leq\left(\int_{\sigma B}|u|^{t} w^{t / s} d x\right)^{1 / t}\left(\int_{\sigma B}\left(\frac{1}{w}\right)^{m t /(s(t-m))} d x\right)^{(t-m) / m t} \\
& \leq\|1 / w\|_{m t /(s(t-m)), \sigma B}^{1 / s}\left(\int_{\sigma B}|u|^{t} w^{t / s} d x\right)^{1 / t} .
\end{aligned}
$$

By the choice of $m$, we find that $r-1=s(t-m) / m t$. Since $w \in A_{r}$, we then obtain

$$
\begin{aligned}
& \|w\|_{1, B}^{1 / s} \cdot\|1 / w\|_{m t /(s(t-m)), \sigma B}^{1 / s} \\
& \quad=\left(\left(\int_{B} w d x\right)\left(\int_{\sigma B}\left(\frac{1}{w}\right)^{m t /(s(t-m))} d x\right)^{s(t-m) / m t}\right)^{1 / s} \\
& \quad \leq\left(|\sigma B|^{1+s(t-m) / t m}\left(\frac{1}{|\sigma B|} \int_{\sigma B} w d x\right)\left(\frac{1}{|\sigma B|} \int_{\sigma B}\left(\frac{1}{w}\right)^{1 /(r-1)} d x\right)^{r-1}\right)^{1 / s} \\
& \quad \leq C_{5}|B|^{1 / s+1 / m-1 / t} .
\end{aligned}
$$

From (3.6), (3.7) and (3.8), we have

$$
\begin{aligned}
\|u\|_{s, B, w} & \leq C_{4}|B|^{-1 / m}\|w\|_{1, B}^{1 / s} \cdot\|1 / w\|_{m t /(s(t-m)), \sigma B}^{1 / s}\left(\int_{\sigma B}|u|^{t} w^{t / s} d x\right)^{1 / t} \\
& \leq C_{6}|B|^{1 / s-1 / t}\left(\int_{\sigma B}|u|^{t} w^{t / s} d x\right)^{1 / t} .
\end{aligned}
$$

It is easy to see that (3.9) is equivalent to (3.2). This ends the proof of Theorem 3.1 .

\section{REFERENCES}

[1] J. M. Ball, Convexity conditions and existence theorems in nonlinear elasticity, Arch. Rational Mech. Anal. 63 (1977), 337-403. MR 57:14788

[2] J. M. Ball and F. Murat, $W^{1, p}$-quasi-convexity and variational problems for multiple integrals, J. Funct. Anal. 58 (1984), 225-253. MR 87g:49011a

[3] S. Ding, Weighted Hardy-Littlewood inequality for A-harmonic tensors, Proc. Amer. Math. Soc., 125 (1997), 1727-1735. MR 97i:30030

[4] S. Ding and C. A. Nolder, $L^{s}(\mu)$-averaging domains and their applications, preprint.

[5] J. B. Garnett, "Bounded Analytic Functions", New York: Academic Press, 1970. MR 83g:30037 (1981 edition)

[6] J. Heinonen, T. Kilpelainen and O. Martio, "Nonlinear potential theory of degenerate elliptic equations", Oxford, 1993. MR 94e:31003 
[7] T. Iwaniec, $p$-harmonic tensors and quasiregular mappings, Annals of Mathematics, 136 (1992), 589-624. MR 94d:30034

[8] T. Iwaniec and A. Lutoborski, Integral estimates for null Lagrangians, Arch. Rational Mech. Anal., 125 (1993), 25-79. MR 95c:58054

[9] T. Iwaniec and G. Martin, Quasiregular mappings in even dimensions, Acta Math. 170 (1993), 29-81. MR 94m:30046

[10] C. A. Nolder, Hardy-Littlewood theorems for A-harmonic tensors, Illinois J. of Math., to appear.

[11] C. A. Nolder, A characterization of certain measures using quasiconformal mappings, Proc. Amer. Math. Soc., Vol. 109, 2 (1990), 349-456. MR 90i:30034

[12] C. A. Nolder, A quasiregular analogue of theorem of Hardy and Littlewood, Trans. Amer. Math. Soc., Vol. 331, 1 (1992), 215-226. MR 92g:30026

[13] S. G. Staples, $L^{p}$-averaging domains and the Poincare inequality, Ann. Acad. Sci. Fenn, Ser. AI Math. 14 (1989), 103-127. MR 90k:42024

[14] B. Stroffolini, On weakly A-harmonic tensors, Studia Math., Vol. 3, 114 (1995), 289-301. MR 96k:58203

Department of Mathematics and Statistics, University of Minnesota at Duluth, DuLUTH, Minnesota 55812-2496

E-mail address: sding@d.umn.edu

Current address, after 9-1-99: Department of Mathematics, Seattle University, Seattle, Washington 98122 